\title{
Filigrane
}

Écoutes psychanalytiques

\section{Actualités de la clinique institutionnelle en France : entre résistance et création?}

\section{Didier Drieu et Pascal Crété}

Volume 24, numéro 2, automne 2015

Le devenir de la psychanalyse. Que nous disent les pratiques institutionnelles?

URI : https://id.erudit.org/iderudit/1036528ar

DOI : https://doi.org/10.7202/1036528ar

Aller au sommaire du numéro

Éditeur(s)

Revue Santé mentale au Québec

ISSN

1192-1412 (imprimé)

1911-4656 (numérique)

Découvrir la revue

Citer cet article

Drieu, D. \& Crété, P. (2015). Actualités de la clinique institutionnelle en France : entre résistance et création ? Filigrane, 24(2), 13-30.

https://doi.org/10.7202/1036528ar

\section{Résumé de l'article}

La psychothérapie institutionnelle a contribué à transformer le monde asilaire en psychiatrie. Elle s'appuie sur la reconnaissance de la psychanalyse, le développement des approches groupales et éducatives actives. L'institution est pensée comme soignante par le mode de relations entre soignants et sujets accueillis et par la création d'un réseau aux prises avec le quotidien et dans lequel émergent des évènements à valeur sémaphorique ou métaphorique. La proximité et les alternatives à l'hospitalisation sont alors des atouts pour retisser des liens avec l'environnement que la politique psychiatrique des années 80/90 est venue mettre à mal, excepté en pédopsychiatrie où les familles ont été de plus en plus associées. 


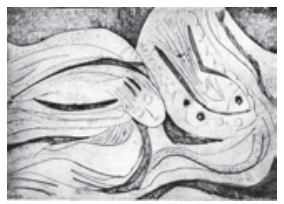

\title{
Actualités de la clinique institutionnelle en France: entre résistance et création?
}

\author{
Didier Drieu et Pascal Crété
}

\begin{abstract}
La psychothérapie institutionnelle a contribué à transformer le monde asilaire en psychiatrie. Elle s'appuie sur la reconnaissance de la psychanalyse, le développement des approches groupales et éducatives actives. L'institution est pensée comme soignante par le mode de relations entre soignants et sujets accueillis et par la création d'un réseau aux prises avec le quotidien et dans lequel émergent des évènements à valeur sémaphorique ou métaphorique. La proximité et les alternatives à l'hospitalisation sont alors des atouts pour retisser des liens avec l'environnement que la politique psychiatrique des années $80 / 90$ est venue mettre à mal, excepté en pédopsychiatrie où les familles ont été de plus en plus associées.
\end{abstract}

ien avant les lois de 2002 et de 2005 qui préconisent de mettre le malade au centre du dispositif de soins, la psychothérapie institutionnelle (PI) a toujours tenté de développer un accueil personnalisé, singulier, au-delà des symptômes présentés. Le patient puise dans l'environnement de l'institution, en son sein et en dehors de ses murs, dans la cité, les moyens de construire son itinéraire, son projet thérapeutique. L'informel, les espaces interstitiels sont alors aussi essentiels au soin que les espaces-temps véritablement structurés. Ils sont des lieux d'échanges où se jouent des rencontres singulières, des relations transférentielles inédites.

Les nouvelles organisations et les mutations sociétales actuelles sont dommageables à ces approches: réductions des lits dans le sanitaire et le médicosocial, développement des soins et de l'accompagnement à domicile, gestion des flux, conception hygiéniste et nosographique de la santé mentale, évitement des risques, protocoles et procédures, dissociation entre le soin et l'éducatif, précarisation des publics... Face à ces nouveaux enjeux, la clinique institutionnelle doit inventer de nouvelles formes, de nouvelles institutions qui répondent aux enjeux de demain. 
Le mouvement institutionnel en France dépasse l'approche de la psychothérapie institutionnelle; il est né dans le contexte particulier de la Seconde Guerre Mondiale et a métamorphosé le travail éducatif et de soin psychique, en recentrant l'accompagnement, l'intervention sur le sujet (patient ou usager) dans son environnement.

Dans le cadre de cet article, nous proposons de revenir sur l'histoire des fondements de la clinique institutionnelle, de discuter des pratiques sousjacentes, de leurs portées principalement dans le soin psychiatrique et de leurs limites face aux profondes mutations qui bouleversent les méta-cadres des institutions.

\section{Les fondements des soins institutionnels: au carrefour du politique, de la psychiatrie et des diverses approches de la psychanalyse}

Remontant historiquement à Pinel et Pussin, le mouvement institutionnel dans la psychiatrie s'est constitué réellement dans l'immédiat aprèsguerre, en résonance avec le drame humain de la guerre (disparition de plus de 40000 malades mentaux), les conséquences négatives du monde asilaire, en opposition à une approche hygiéniste de la maladie mentale et en référence à différentes influences. Bien sûr, une première source majeure vient de la psychanalyse et la psychiatrie clinique au milieu des années 40, avec l'expérience de François Tosquelles, psychiatre catalan réfugié à Saint-Alban. Celui-ci a initié le mouvement de la psychothérapie institutionnelle en s'appuyant à la fois sur son expérience politique, celle d'un militantisme du $\mathrm{POUM}^{1}$, et en s'inspirant de la démarche d'Hermann Simon, psychiatre allemand, relative à l'importance d'associer les malades à l'amélioration du cadre hospitalier et à son engagement pour la psychanalyse (travaux de Freud et premiers écrits de Lacan). Signalons aussi l'apport de Georges Daumézon (Fleury les Aubrais) qui, avec l'aide du mouvement associatif des CEMEA ${ }^{2}$, organise la formation des professionnels à la psychiatrie et aux techniques de groupe. Il initie aussi le Groupe de Sèvres où participent Tosquelles, Oury, Bonnafé... qui mène avec les soignants une réflexion sur la logique asilaire, ses hiérarchies et ses modes d'aliénation, de chronicisation, et sur certaines formes de dépendance extrême et de liens pervertis entre les différents acteurs de l'asile. Enfin, ces pionniers utilisent d'autres références comme l'apport des expériences des phénomènes de groupe des auteurs anglo-saxons (Harry Stack Sullivan, Kurt Lewin, Jacob Lévy Moreno), des techniques d'éducation active de la pédagogie institutionnelle (Célestin Freinet, Fernand Oury, 
Alexander Sutherland Neill, Fernand Deligny) comme le montre Jacqueline Barus-Michel.

Cette expérience de militantisme d'après-guerre va s'associer aux projets politiques des gouvernements de la IVe République dans les années 60 avec les premiers textes modifiant considérablement l'univers psychiatrique hospitalier. Elle va rencontrer aussi Henry Ey, fondateur de l'approche organo-dynamique, créateur de la revue et de la société de l'Évolution psychiatrique, qui contribuera à établir des ponts entre les cercles psychanalytiques et la psychiatrie jusqu'alors peu ouverte aux notions psychanalytiques. Ces liens vont amener un groupe de pionniers à organiser des débats pendant plusieurs années (Groupe de Sèvres, puis GTPSI ${ }^{3}$, puis Société de Psychothérapie Institutionnelle) qui porteront à la fois sur la politique de la sectorisation (évolution de l'hôpital psychiatrique, formation des internes et des infirmiers en psychiatrie, création des hôpitaux de jour, soins à domicile, relation médecin/malade...) et la participation des infirmiers aux psychothérapies. Les débats sur la psychothérapie vont provoquer une scission entre les "psychiatres psychanalystes», souhaitant développer la psychothérapie institutionnelle et ceux comme Philippe Paumelle, Jean Kestemberg et René Diatkine qui considèrent que la place de la psychanalyse doit être pensée dans la différenciation des fonctions, des cadres, des espaces.

Aussi, une première approche du soin psychique en institution s'est formée autour des apports de François Tosquelles, celle de la psychothérapie institutionnelle et de ses prolongements — «dans la pédagogie telle que l'expérience de l'école ouverte de Célestin Freinet, à la philosophie (JeanPaul Sartre, Cornélius Castoriadis), à l'intervention psychosociologique et sociologique avec les idées de Georges Lapassade, René Lourau» (Drieu, 2013, p. 4), voire Max Pagès, Vincent De Gaulejac. Jacqueline Barus-Michel (1987) a mis en évidence les liens que l'on pouvait établir entre ces différents courants et la psychologie sociale clinique. Au niveau du travail des équipes de soins, les acteurs du soin seront soucieux de reprendre «les évènements dans les pratiques, de prendre en compte ce qui ne va pas de soi, les agirs et manifestations de la chronicité des patients psychotiques par exemple, leur évolution et ainsi «entrer en résonance avec leur fonctionnement psychique» (Jean Oury, 1986). L'institution dans son quotidien doit être thérapeutique en elle-même et le travail clinique est centré sur les enjeux inconscients institutionnels. L'élaboration s'appuie sur le travail de «l'accueil» qui, comme nous le développerons plus loin, ne se limite pas à une observation à l'entrée du sujet accueilli, mais se prolonge au niveau d'une attitude du 
collectif organisé par le tissu des réunions: «la fonction phorique» collective chez Pierre Delion, support d'une réflexion sur la « constellation transférentielle» dans laquelle sont pris soignants et patients (1999). De même, on accorde une attention particulière aux activités sociales, à la participation des malades ou usagers au club thérapeutique, aux associations culturelles, objets d'investissement œuvrant à la refondation d'une vie sociale, d'investissements plus ouverts. La fonction thérapeutique à travers une activité «sémaphorique» et "métaphorique» peut être partagée par tous les acteurs de l'institution à la condition que s'exerce un «fonctionnement diacritique du collectif» fonction qui introduit une logique castrative (Delion, 1999). «Si cette expérience s'est diffusée dans la psychiatrie et dans certains services médico-sociaux jusqu'aux années 80 , son idéal auto-gestionnaire s'est très vite heurté aux cloisonnements de la sectorisation et aux mutations profondes de l'organisation des soins psychiatriques» (Drieu, 2013, p. 4).

Une autre approche du travail de soin psychique s'est formée autour de psychanalystes qui souhaitaient associer plusieurs registres complémentaires dans l'accompagnement thérapeutique: la dimension psychothérapeutique avec celle de la référence médicale, de l'éducatif ou de la réhabilitation psychosociale. Dans ce contexte, la clinique institutionnelle peut associer plusieurs fonctions essentielles. D’une part, afin de soutenir une présence active des soignants et autres intervenants dans l'accueil au sein de l'institution. Et d'autre part, dans le but de mettre en perspective des repères dans différents espaces de soin permettant de symboliser scansion et continuité par rapport à l'accompagnement du patient dans ses différentes phases. "Il s'agit plus alors de penser avec l'institution telle qu'elle se présente sans prétendre la transformer. Le soignant, l'intervenant social s'intéresse plus à la réalité de ce que vit le patient; le psychothérapeute ou psychanalyste se situant davantage dans une clinique psychodynamique (psychothérapies, médiations thérapeutiques)»(Drieu, 2013, p. 4).

Toutefois, face à des patients psychotiques, Paul-Claude Racamier, par exemple, n'hésite pas à parler des soignants dans une institution comme des ambassadeurs de la réalité. Ceux-ci jouent le rôle de contenant des angoisses psychotiques projetées en s'appuyant sur les pratiques courantes et un mode de rêverie partagée afin de soutenir une activité de symbolisation. À partir de son expérience du foyer psychothérapeutique de la Velotte à Besançon, il évoquera ainsi un travail sur plusieurs scènes institutionnelles. Ces démarches sont celles de Philippe Paumelle et René Diatkine dans le secteur du $13^{\mathrm{e}}$ arrondissement, précurseurs du mouvement de sectorisation 
en mettant l'accent sur la proximité mais surtout sur le soin de préférence à l'assistance. Si l'expérience analytique, le transfert relève de la cure-type et de ses aménagements, le travail thérapeutique doit pouvoir être associé par exemple à la consultation ou à des médiations, à l'accompagnement, la prise en charge prenant alors la forme de la bi-focalité ou plurifocalité. Ils insistent beaucoup sur la différenciation des espaces de référence au risque de voir l'équipe confrontée à une écoute à vif des contenus inconscients sans cadre d'interprétation, comme le souligne René Diatkine (1970). «Au contraire, dans l'esprit des psychiatres, psychanalystes s'inspirant du courant de la psychothérapie institutionnelle, le transfert concerne tout le monde et doit donc être mis au travail dans des espaces de paroles institués » (Drieu, 2013, p. 5).

Quelle que soit l'approche envisagée, ces collègues ont dû s'intéresser aux fonctionnements de groupe, d'où un intérêt pour les travaux de la psychosociologie et aujourd'hui de l'intersubjectivité initiés par Donald Winnicott et Wilfred Ruprecht Bion. En France, si l'initiative d'une réflexion sur «le groupe et l'inconscient» revient à Didier Anzieu en 1975, la conceptualisation d'une réflexion sur «les effets de l'inconscient dans les institutions», tant soignantes, éducatives que sociétés savantes de psychanalyse, s'est faite sous la direction de René Kaës (1988). L'idée était alors de «rendre compte des forces contraires qui travaillent l'institution: les unes œuvrent à unifier, essentiellement grâce au développement de l'idéal, des représentations de la cause unique, des synergies d'investissement libidinal; les autres travaillent à la différenciation et à l'intégration des éléments distincts dans des unités de plus en plus grandes; d'autres au contraire sont vouées au retour de l'indifférencié, à la réduction des tensions; d'autres encore à la destruction et à l'attaque» (p. 16-17). Dans ce livre princeps, se trouve alors ébauchée «la question des nouages des alliances négatives» qui participent aux crises, à l'escalade dans les souffrances des équipes, aux mouvements de fondation ou refondation des instances institutionnelles dans les mutations (changements de direction, du public accueilli, des normes), à la construction des mythes étayant la maintenance du contrat narcissique mais aussi, les silences sur l'histoire passée (professionnels et personnes accueillies).

Dès lors, en référence à ces approches, comment pouvons-nous décrire les principes du soin institutionnel, la créativité de ses diverses instances et ses limites? 


\section{Les principes du soin institutionnel: transformer le milieu}

Les soins psychiques "ne peuvent se faire que dans des institutions créées entre les soignants et les sujets accueillis », là où il existe des espaces de rencontres, d'élaboration mais aussi des lieux de projection de conflits d'abord vécus, contenus, puis figurés dans les réunions (Delion, 2006, p. 13). Ce réseau, dans une institution aux prises avec le quotidien, doit pouvoir donner lieu à des reprises permettant la transformation des évènements observés (la dimension sémaphorique) en scénarios psychiques figurables (la dimension phorique), autant d'intermédiaires vers un accès à une activité de symbolisation. Selon Jacques Hochmann (1982, p. 990), «le soin collectif consiste à [lui] offrir un contenant actif, un transformateur d'émotions brutes en sentiments, un lieu psychique où les éprouvés corporels ou mentaux du patient trouvent un sens, à travers l'investissement dont il est l'objet de la part du soignant». Tributaire de la sortie des malades hors des murs de l'asile (le désaliénisme), un des premiers objectifs du soin institutionnel était de travailler le lien social, le rapport à l'environnement.

\section{La sectorisation, les soins de proximité}

Dans le prolongement de la psychothérapie institutionnelle, la sectorisation psychiatrique, là où elle a pu se construire, s'est organisée autour de la création de dispositifs de soins de proximité, des lieux ouverts, bien repérés dans la cité. Tout en étant moins radicale qu'en Italie, il s'agissait de sortir de l'espace asilaire en mobilisant «les capacités de création collective et d'entraide pour favoriser l'instauration de liens, pour accueillir la souffrance ou la détresse psychique quel que soit le lieu et la forme de son expression, sans a priori et sans préalable» (Oury et Orange, 2013, p. 155). Après parfois un long temps de chronicisation ou des violences survenues à l'occasion d'une crise, il faut bien sûr prendre le temps nécessaire pour rétablir un lien de confiance réciproque entre le sujet malade et son environnement, les membres de sa famille. Ainsi, en psychiatrie adulte, l'enjeu sera de penser la réinsertion, puis plus tard, la réhabilitation dès l'entrée dans le service afin «de limiter les effets d'exclusion du droit commun et à la citoyenneté pour les personnes les plus vulnérables» (Oury et Orange, 2013, p. 155). Cependant, même si les grands principes de la sectorisation (proximité, accessibilité, décloisonnement) sont toujours d'actualité en psychiatrie adulte, ils se sont souvent retrouvés contrecarrés par des logiques hospitalières nouvelles (Loi HPST ${ }^{4}$ et organisation en Pôles d'activité), de suppression de moyens, d'hyperspécialisation provoquant une sorte 
d'éclatement des repères des équipes abandonnées par des psychiatres trop souvent enfermés dans des fonctions d'expertise. Progressivement, les initiatives reviennent par le biais des associations d'usagers, «de porteurs de Groupes d'Entraide Mutuelle, de Résidences Accueil, de clubs d'expériences d'insertion professionnelle innovante, de services d'accompagnement à la vie sociale. [...] Les psychiatres et les équipes sont obligés actuellement, sous l'impulsion des usagers, de repenser leur place, de reprendre en compte la notion de vulnérabilité, de questionner le "prendre soin" qui va au-delà d'une vision technique du soin médicalisé» (Oury et Orange, 2013, p. 155).

Le cheminement est quelque peu différent du côté des intersecteurs de pédopsychiatrie ou dans les associations (ASM 13 ${ }^{5}$, Fondation Vallée 6 , $\mathrm{CMPP}^{7}$ ) porteurs de projets de soins relativement à des enfants, des adolescents longtemps stigmatisés. Les positionnements de Roger Misès par rapport à la fondation Vallée, de René Diatkine par rapport à l'ASM 13 et, même de Maud Mannoni par rapport à l'école ouverte de Bonneuil sur Marne illustrent les débats forts sur cette notion de soins de proximité. Pour eux, il s'agissait bien de lutter contre l'approche d'une psychiatrie interniste qui renvoyait les enfants en situation de handicap grave à l'image de l'arriération mentale, ou encore à celle de la déficience, voire l'idée d'incasable pour les adolescents présentant des problématiques narcissiques graves, et ce, en organisant des soins intégrant à la fois les enjeux éducatifs et psychiques de l'enfant. Cependant, pour les deux premiers, la cure doit être «multidimensionnelle», s'appuyant sur un réseau de "médiations psychothérapeutiques», sur des "tiers référents» et sur un travail avec les éducateurs, les écoles, les familles, avec l'objectif de «remettre en route l'imaginaire et le fonctionnement préconscient à partir d'une expérience concrète dans un espace d'illusion» (Chagnon, 2013, p. 102). Pour Maud Mannoni, malgré la représentation d'une institution éclatée, multiréférencée, l'approche de l'enfant se modélise davantage sur la subversion d'un modèle, celui de l'éducation ou de la rééducation trop tournée vers une «image d'enfant idéal» qui interdit l'imprévu, rend passif l'enfant (De La Jonquière, 2013). Toutefois, les deux approches sont beaucoup moins antagonistes aujourd'hui si nous nous référons aux travaux de Pierre Delion qui montrent bien l'importance de synthétiser «les deux sens de la psychothérapie institutionnelle: construire toute l'institution comme espace thérapeutique potentiel et faire la thérapie de l'institution, l'un et l'autre étant indissociablement liés» (Robin, 2013, p. 163-164).

Progressivement, le soin s'est élargi vers une ouverture à l'environnement comme dans le partenariat avec les Réseaux d'aide à l'école (RASED), 
mais surtout l'intégration des parents, des familles dans le processus psychothérapeutique. Au départ, ces dynamiques de soins ont été élaborées pour prévenir les intrusions, les ruptures, afin de devenir par la suite de véritables espaces thérapeutiques élargis, là où le travail de soin auprès d'un enfant s'accompagne d'un travail avec la famille comme dans les prises en charge plurifocales, les groupes parents-enfants.

Pour soigner des sujets en souffrance psychique, il a été indispensable de réfléchir aux dispositifs nécessaires pour les accueillir, pour les accompagner dans le quotidien de l'institution et dans un processus thérapeutique.

\section{La psychothérapie institutionnelle et l'accueil}

Dans le monde psychiatrique hospitalier, l'accueil reste trop souvent centré sur l'examen, l'évaluation des troubles, le sujet malade étant perçu comme un «objet de connaissance» présentant des symptômes qu'il faut pouvoir évaluer pour les traiter. Même si les nouvelles références sur les droits des malades ont pu faire évoluer ces pratiques vers une meilleure information, le patient reste pris dans cette logique du savoir médical. Même dotée d'une ouverture vers des approches complémentaires comme l'anthropologie, le droit, la psychanalyse, le soignant porteur de cette aspiration risque de manquer la rencontre propice à une construction d'un univers de sens pour le sujet malade. Il y a souvent confusion entre l'admission, inscription sur le registre des entrées, et l'accueil, qui relève d'une disposition psychique. «Être-là» et accueillir l'autre tel qu'il se présente.

La «révolution culturelle» qu'a tenté d'opérer le courant de psychothérapie institutionnelle porte sur l'idée de mettre le malade au centre non seulement des soins mais aussi «de sa guérison» (Delion, 2006). Cela rejoint l'idée chère à Jean Oury: «L'hôpital devrait être hospitalier»(Oury, 2001). L'hospitalité psychiatrique consiste donc à accueillir tout patient, même pris dans des fonctionnements insolites, d'une façon non traumatisante, en établissant constamment avec lui des rapports les plus authentiques possibles. Or, très souvent, la rencontre avec le malade se fait au moment le plus compliqué, dans l'urgence. Il faut donc travailler avec les soignants une fonction d'accueil qui permette de rencontrer l'autre en déshérence psychopathologique dans de bonnes conditions, soigner son accueil. Ainsi, s'agira-t-il souvent de faire en sorte d'accueillir en respectant le cheminement du sujet, ce qui veut dire que le dispositif/cadre de l'accueil puisse faire l'objet de discussions, de négociations, afin de favoriser le partage, la construction d'un lien, mais surtout dans le but de tenir compte de l'investissement d'un sujet 
comme préalable à la relation thérapeutique. L'accueil, tel que nous pouvons en rendre compte dans cette expérience de la psychothérapie institutionnelle, est alors une ouverture à l'autre avec l'idée forte de faire une place à la demande du patient, de le recevoir comme un autre humain qui attend d'être accueilli en dépit de tout préjugé lié à sa maladie. Ainsi, à la clinique de La Borde, le futur entrant est invité à visiter la clinique avec un comité d'accueil, les «poissons-pilotes»: des pensionnaires qui, tout en lui faisant visiter les lieux, l'informent de la vie, telle qu'eux la vivent, à la clinique (Oury, 1990).

Cependant, nous comprenons dès lors que ce souci de l'accueil ne s'arrête pas au sas d'entrée mais qu'il doit être constamment porté par le collectif. Ainsi, «après François Tosquelles, Jean Oury interroge l'institution dans laquelle les soins se conjuguent (hôpital, service, foyer...). Peut-elle être un milieu suffisamment "travaillé collectivement" par des outils spécifiques afin de produire une fonction d'accueil et soignante?» (Crété, 2013, p. 138). Aussi, l'accueil dans cette approche ne peut être un acte individuel, le colloque singulier médecin/malade par exemple, il est au contraire un acte collectif, même si en psychiatrie (ou santé mentale) la place du médecin est importante. De même, l'accueil ne se borne pas à l'admission, mais se prolonge dans tous les rapports du sujet accueilli avec l'institution. Cependant, il est nécessaire que les soignants (ou intervenants) puissent être à l'écoute des rebondissements, des résonances du vécu de l'accueil dans les évènements quotidiens, qu'ils puissent s'en saisir pour en donner une lecture sensible, selon «sa constellation transférentielle» (Delion, 2006, p. 14). Ailleurs, dans une approche plus différenciée de la clinique institutionnelle, comme par exemple un intersecteur de pédopsychiatrie, Jacques Hochmann parle d'une institution centrée sur le patient, avec l'idée de construire avec et autour de lui « un cadre malléable constitué de la réunion de tous les interlocuteurs du patient (psychiatre, infirmier, assistante sociale, employeur, etc.), quels que soient leurs services d'appartenance, autour d'un projet commun» (Chagnon, 2013, p. 102). Ainsi s'agit-il de construire entre l'enfant, sa famille, l'école, la cité et les soignants une histoire commune, scandée par la temporalité, la différenciation des espaces, et organisée par la dimension instituante du collectif institutionnel.

Cette notion d'accueil s'articule avec la nécessité d'une ambiance permettant des espaces différenciés, non réductibles l'un à l'autre et pouvant susciter pour le patient de réelles possibilités de choix, d'investissements, et ainsi produire des effets positifs en laissant émerger les éléments de son psychisme. 


\section{Des espaces d'investissement différenciés et ordonnés pour la continuité des soins}

Hermann Simon, psychiatre allemand de l'hôpital de Wartein (Asile de Guttersloch), précisait déjà en son temps que pour chaque patient, il était nécessaire de s'intéresser aux «parties saines» du psychisme, souvent négligées par le psychiatre (Simon, 1933). Percevant l'hôpital, l'asile comme un organisme malade, il voulait lutter contre l'inaction, l'ambiance défavorable des services et les projections des « soignants ", à savoir le préjugé d'irresponsabilité. Il soutenait l'idée que le patient peut trouver des sources d'investissement dans l'environnement de l'hôpital, ce qui deviendra des activités de groupe, des moyens psychothérapeutiques pour mobiliser le sujet malade conditionné par l'ambiance des lieux. Cette idée du soin dépend beaucoup des rapports entre l'organisation (l'établissement) et l'institution (le collectif) qui peuvent susciter une culture institutionnelle avec des logiques antagonistes mais en même temps, bien complémentaires. La notion d'ambiance renvoie aux phénomènes de transfert qui touchent profondément le devenir de ces groupes et sujets, une constellation transférentielle qu'il y aura nécessité d'analyser en permanence afin d'éviter la chronicisation et les projections. Ainsi s'agit-il, dans l'esprit du secteur psychiatrique, de créer des liens entre l'intra et l'extra hospitalier afin de permettre la sortie des patients hors des murs bien sûr mais bien au-delà, de "créer une "mosaïque" permettant de multiples "itinéraires", "itinéraires pulsionnels", "à la carte"» (Chaperot et Celacu, 2008, p. 450).

Ainsi, au-delà de la différenciation des lieux (services d'hospitalisation temps plein, hospitalisation de jour, centre médico-psychologique, Centre d'Activité Thérapeutique à Temps Partiel...), l'idée a été de travailler sur l'ambiance du soin psychiatrique. La logique des secteurs a été d'instaurer des structures ouvertes à la liberté, aux allées et venues mais aussi des points de repères sur lesquels les relations peuvent s'établir. Ces espaces de rencontres seront constitués au départ par le club thérapeutique soutenu par une association et les ateliers qui vont s'initier à l'intérieur et à l'extérieur des murs de l'hôpital. Leur mise en place fait l'objet de débats, ce collectif ayant déjà une première fonction soignante, la fonction phorique, de holding. Dans les institutions médico-sociales, de même, il a été question de rompre avec la philosophie interniste en s'appuyant sur une différenciation des lieux d'investissement des enfants (école, ateliers), de l'éducatif et du thérapeutique (psychothérapies à l'extérieur), des espaces de projets et de vie (familles d'accueil, ambulatoire). 
Les ateliers sont devenus alors autant de lieux de support à la relation où se jouent à la fois les désirs et les fantasmes du patient, mais aussi des conflits permettant que se relance «l'investissement du patient par autrui en même temps que son propre sentiment d'existence» (Azoulay, 2004, p. 10). Ces activités, d'abord modelées sur les principes de l'ergonomie, l'occupationnel dans le monde asilaire, se sont progressivement élaborées autour d'un projet thérapeutique avec l'idée d'une liberté de s'investir, de circuler dans les activités. Souvent proposée en dehors des murs de l'hôpital, la circulation entre le dedans et le dehors, dans les ateliers, met les patients en situation de se réapproprier un horizon temporel, des modalités de choix, autant de repères leur permettant d'éviter paradoxalement l'installation d'une chronicité linéaire. Il n'y a plus de «temps maladie» balisé par un contrat de soin unique, celui de la psychiatrie interniste, mais un temps à la fois discontinu par la suspension, la fin de certains liens, et continu quand nous considérons la prise en charge globale. De plus, chaque participant peut se confronter à la question de son désir d'investir tel lieu, telle activité, d'y revenir ou non, accompagné dans ses interrogations par d'autres. Même si pour certains ateliers (groupes ouverts) il n'y a pas d'engagement thérapeutique, les échanges permettent d'élaborer l'absence, les moments de vide, ce qui n'est pas sans produire des effets thérapeutiques à long terme. Le désir et l'engagement des soignants comptent beaucoup pour produire des effets symboliques car ils viennent en apportant de l'extérieur (le non Moi) leurs propres investissements (Moi), installant de ce fait une sorte d'ambigüité, un fonctionnement transitionnel créateur qui permet, soutenu par l'ambiance, des formes d'expression sans cesse renouvelées et un soutien constant auprès des patients. Winnicott situe la créativité dans ce champ des expériences transitionnelles, entre la réalité interne et celle du monde extérieur, et insiste sur sa fonction principale: celle de la découverte de soi et plus tard, celle de retrouver la confiance dans sa continuité, dans les liens, dans sa capacité de jouer, de créer. Toutefois, face à la vulnérabilité des patients, cette continuité risque d'être attaquée en permanence. C'est pourquoi il s'agit bien "d'instituer alors des dispositifs venant soutenir à la fois une continuité et une discontinuité dans le travail clinique et par de là, offrir des médiations permettant de traiter la question de la dépendance par rapport aux aidants » (Winnicott, 1958, cité par Drieu, 2013, p. 284). 


\section{Le travail clinique institutionnel: de l'observation dans les interstices au partage dans le collectif soignant}

Cependant, à côté de ces fonctionnements institués, il y a la nécessité d'être à l'écoute de l'informel, ce qui va se développer dans les espaces d'entre-deux:

[ces] fonctionnements institutionnels atypiques (atopiques, utopiques?) — interstitiels — dans lesquels se localise ce qui n'est pas inscriptible ailleurs. Nous retrouvons beaucoup ces fonctionnements dans les observations fines de certains professionnels, souvent ceux qui vont partager le quotidien des patients comme les agents hospitaliers, ou les «maitresses de maison» avec les usagers, les «travailleuses familiales» qui accompagnent les parents dans les espaces de médiation famille. Ces processus groupaux peuvent venir doubler des processus structurés, prenant appui ou contreappui sur ceux-ci, ou au contraire, s'insinuer entre les espaces-temps institutionnels structurés (Roussillon, 1988, p. 159).

Quelles que soient nos approches face aux institutions, la psychanalyse groupale, celle des méta-cadres, la psychothérapie institutionnelle, nous retrouvons des réflexions autour de cette question des interstices, des corridors chez Paul Fustier (1993, 2012), voire de l'informe s'inscrivant dans les fonctionnements de l'accueil, de l'accompagnement dans les soins psychiques (Delion, 2011). Dans ce contexte, la clinique informelle, c'est-à-dire tout ce qui vient des observations, liens initiés dans les corridors, couloirs, compte beaucoup, avec comme corollaire les investissements des différents acteurs, la manière dont l'institution peut en réguler sa portée. Ainsi se posent des questions comme: "l'interstice est-il un lieu de travail ou un espace privé? La valeur régulatrice de l'interstice institutionnel est suspendue à l'indécidabilité de cette question» (Roussillon, 1998, p. 169). À quel moment formalisons-nous un type d'échange d'abord et avant tout informel. Nous pensons aux débats infinis sur le repas thérapeutique, le séjour thérapeutique, etc. L'ambiguïté de l'interstice, nous dit Roussillon, permet en effet des aménagements de la distance subjective à l'autre qui rendent possible de demander à tel ou tel de "tâter le terrain " concernant un problème donné. Elle évite ainsi des dommages narcissiques, réels ou fantasmés, d'une parole qui prendrait le risque de se faire entendre et d'essuyer rejet ou refus (Roussillon, 1998, p. 169). Lieux de dépôt mais aussi de mise en latence de l'informe, ces objets ou espaces de projection peuvent avoir un 
caractère organisateur par rapport à la vie institutionnelle dans la mesure où ils viennent en renforcer les processus d'élaboration.

Le travail clinique collectif, nous dit René Roussillon, consistera à reconnaître ce qui se joue dans ces espaces, puis au-delà, à pouvoir élaborer ce qui se joue pour les différents acteurs. Parallèlement, on retrouve dans une approche un peu différente la même perspective chez Pierre Delion quand il insiste sur l'idée d'être dans les activités et ses entre-deux dans une posture sémaphorique: être observateur de mouvements psychiques naissant dans l'informe, l'informel, des observations à partager sur la scène institutionnelle, à rendre figurables (métaphorisation) avant d'aller vers le symbolique. Cette construction à plusieurs soignants constitue en quelque sorte la feuille d'assertion, le lieu sémaphorique de ce qui est à interpréter. C'est dans le mouvement transfert-contre-transfert qu'elle va apparaître (Delion, 2001). La feuille d'assertion est comme une substance malléable qui se déforme pour être au plus près de ce qui vient des sujets accueillis mais sans se répandre, s'enkyster, à condition d'être reconnue au niveau du collectif. C'est pourquoi le dispositif institutionnel (l'organisation) doit soutenir l'ambigüité de l'interstice: l'expérimentation, le jeu des échanges dans les réunions et en dehors. Travailler avec l'interstitiel, dans l'informel nous renvoie à la nécessité de prendre appui sur l'expérience des membres du groupe, d'analyser leur implication, de recourir à l'intervention psychodramatique (une pensée imagée, métaphorisante).

\section{Les mutations dans le paysage du soin en santé mentale}

Force est de reconnaittre qu'aujourd'hui cette clinique de l'interstice, de l'informel est en péril dans les services de soin psychiatrique (ou santé mentale) et dans les institutions médico-sociales. Dans les situations de crise, la fonction «débarras» d'une réunion, d'une figure d'un soignant peut se trouver saturée et disqualifiée, empêchant un travail collectif, un consensus groupal sur la reprise de ce qui s'y déroule. Très souvent, cela arrive quand il y a saturation des dispositifs institutionnels tels que des recouvrements traumatiques liés à des changements (changement de médecins, de direction, dans l'accueil des patients...). Cependant, aujourd'hui, c'est à une crise généralisée de la clinique institutionnelle en France à laquelle nous assistons du fait de profondes mutations des métacadres dans les nouveaux Établissements Publics de Santé Mentale, dans le Médico-social (Pinel, 2008, cité par Drieu, 2013, p. 6): 
Globalement, nous sommes passés d'établissements avec une organisation de type charismatique à une forme de management gestionnaire, d'institutions organisées dans des fonctionnements pyramidaux mais complémentaires (les structures héritées de la société patriarcale) à des services de plus en plus modélisés sur des logiques de prestations et donc de concurrence organisée de manière réticulaire. Dans un contexte de rupture de ce qui soutient la fondation du soin, de l'éducatif $[\ldots]$

Nous perdons pied ici avec le processus d'historicisation, indispensable pour la construction des projets dans les institutions, et face au devenir qui se dérobe, les équipes risquent de fonctionner dans le repli, la peur, la défiance, et ce, par manque d'accordage entre processus sociaux et liens intersubjectifs (Drieu, 2013, 6; Kaës, 2012). Ainsi, au-delà de la question des moyens (suppression de plusieurs milliers de lits en psychiatrie), il existe plusieurs registres de mutations profondes. Nous assistons à une révolution dans l'idée même du soin psychique avec le passage radical de la lutte contre les causes et conséquences de la maladie mentale à une conception plus hygiéniste de la santé mentale, transformant les interventions des différents acteurs en procédures d'évitement des risques. Concourant à son éclatement, les frontières entre les mondes du soin et de l'éducatif sont devenues très poreuses avec par exemple la notion de handicap psychique qui semble légitimer davantage les protocoles d'éducation thérapeutique plutôt que des soins psychiques élargis. Si une certaine «porosité» est parfois souhaitable entre les différents domaines, elle prend ici la forme d'une confusion empêchant une complémentarité attendue entre l'éducatif et le soin. Cette situation peut se trouver renforcée par la perte de repères des équipes, tant du côté des connaissances des troubles, avec les nouvelles nosographies (DSM) qui réduisent l'évaluation à des traits objectivables, que du côté des publics accueillis qui présentent souvent des situations empreintes de multiples sources de précarité. Régulièrement viennent se rajouter des problématiques de directeurs ou médecins qui incarnent moins l'autorité — sousentendue la potentialité à tenir une direction, à faire des choix étayant des pratiques institutionnelles —, mais davantage un management centré sur l'économique, la gestion des flux. Nous pouvons également ajouter l'importance d'une crise de la professionnalisation des praticiens de la clinique dans les collectifs de soins avec la perte de la spécialisation psychiatrique chez les infirmiers, la diminution des psychiatres, les crises intergénérationnelles (écarts de pratiques entre les générations), des carences de transmission 
contre lesquelles les dispositifs de tutorat tentent de lutter. L'ensemble de ces faits concourent à recréer des cloisonnements, à figer l'informel, à désarticuler les liens entre les équipes dans des modes de clivage et de concurrence, d'autant plus quand l'organisationnel est dans une recherche de la maîtrise avec des protocoles suscitant de la défiance, comme l'explique René Kaës (2012) dans son livre «Le Malêtre».

Ces souffrances viennent se condenser avec celles des sujets que nous accueillons, des sujets souvent désarrimés dans leurs processus de subjectivation car souffrants, tant du côté de leur base narcissique que de celui d'une appartenance à un ensemble social; des sujets marqués de façon concomitante par la mésinscription des liens et par des violences traumatiques transsubjectives. Face à une dynamique de recouvrement traumatique quand par exemple les usagers ou patients accaparent les professionnels avec un trop plein d'angoisses qui débordent leurs défenses collectives, en situation de recouvrement de tensions, "collapsus de la topique institutionnelle», les interstices peuvent se faire "cryptes», favorisant alors les clivages, des fonctionnements paradoxaux, "une partie officielle, une partie occulte dans l'institution» (Pinel, 2007; Roussillon, 1988, p. 170). Dans ces conjonctures se pose alors le problème des modes de régulation, encore plus aujourd'hui où les secteurs dits de santé mentale (la psychiatrie adulte) affrontent de nombreuses mutations. Face à cette situation de crise, il est nécessaire de penser le cadre de l'intervention à partir de ces systèmes de régulation en mobilisant à la fois la pensée commune (groupes de parole), mais aussi une dimension de «co-construction» du cadre et du processus de l'intervention.

Toutefois, face à ces changements radicaux, des collectifs institutionnels subsistent et se transforment en conservant un terrain propice à la transmission de la clinique, comme dans l'expérience institutionnelle à la clinique La Borde, celle de La Chesnaie, certains secteurs de psychiatrie ou de pédopsychiatrie (p. ex. Landerneau), des lieux du secteur médicosocial (p. ex. Foyer Léone Richet à Caen). Dans un tout autre contexte, celui de l'expérience des ex-patients de la psychiatrie et des Groupes d'Entraide Mutuelle, il s'agit d'œuvrer à une prise de parole des usagers qui permette d'inverser une vision négative du monde social par rapport aux sujets vulnérables, afin de transformer les dynamiques de réhabilitation psychosociale vers davantage d'inclusion. Le Conseil de Santé Mentale peut être un maillon de ce fonctionnement, comme le montre l'évolution du secteur de psychiatrie adulte à Lille avec le Dr Roellandt qui a transformé les unités d'hospitalisation en équipes mobiles, susceptibles d'interagir avec le réseau existant dans la cité 
pour accompagner au plus près les crises avec des hospitalisations à domicile, des pratiques interstitielles dans les lieux culturels, etc. D'autres dispositifs de clinique institutionnelle, comme l'Association de Santé Mentale $\mathrm{du} 13^{\mathrm{e}}$ arrondissement, la clinique Montsouris et le centre Étienne Marcel évoluent en créant des réseaux de recherche-action, en intégrant les nouvelles nomenclatures diagnostiques tout en déconstruisant leurs dimensions désobjectivantes.

\section{Conclusion}

La psychothérapie institutionnelle a entraîné un changement de paradigme transformant l'approche médicale fondée sur le symptôme, le diagnostic et le traitement en une approche plus générale appréhendée à travers le concept d'accueil, ce dernier prenant davantage en considération le sujet dans ses dimensions relationnelles et environnementales. Ainsi, à l'internalisation qui produit l'isolement du patient, elle répond par l'externalisation, l'alternance ou la complémentarité des soins avec une mise à disposition de moyens «intra» et "extra», des ateliers, des clubs, des groupes de parole, comme autant d'occasions pour le sujet de faire lien avec autrui. Les soins strictement psychiques sont inscrits dans un contexte où il s'agit surtout de prendre soin en visant la participation du sujet à sa guérison et l'intégration du sujet dans l'institution, reliant ainsi le soin à des questions relevant habituellement du champ médico-social. Les frontières entre les approches thérapeutiques et socio-éducatives sont cependant maintenues comme distinctes dans l'approche institutionnelle. A contrario, elles peuvent apparaître aujourd'hui comme "poreuses» dans les politiques et réalités hospitalières du moment, du fait non pas d'une approche globale du sujet mais de la prégnance du modèle gestionnaire et de la centration toujours plus affirmée des normes évaluatives, à l'image d'ailleurs du monde de l'entreprise. La psychothérapie institutionnelle peut apparaître ainsi dénaturée de sa fonction première et il faut toute son inventivité créatrice, la mobilisation de ces équipes, pour résister et lutter contre cette déshumanisation.

Il s'agit alors pour les collectifs de soins en santé mentale de faire face à la défiance dans l'horizon du mal-être actuel, de renouer avec la créativité première, de rompre cette fois-ci non plus avec la logique asilaire, ce qui avait inauguré sa démarche, mais au contraire de résister à une logique gestionnaire, qui empêche la prise en considération des différentes dimensions du soin ou qui les amalgame dans un souci d'économie. Il s'agit aujourd'hui comme hier de maintenir une continuité des soins dans l'histoire du sujet 
en s'appuyant sur un réseau de plus en plus dense et ouvert. C'est le défi non seulement d'un avenir pour la psychothérapie institutionnelle, mais des nouvelles formes de professionnalisation pour répondre aux questions de précarité et de violence qui accompagnent de plus en plus souvent les questions strictes de santé mentale.

\section{Didier Drieu didierdrieu@unicaen.fr}

Pascal Crété

\section{Notes}

1. Partido Obrero de Unificación Marxista.

2. Centre d'entraînement aux méthodes d'éducation active.

3. Groupe de travail en psychothérapie et sociothérapie institutionnelles.

4. «Loi portant sur la réforme de l'hôpital et relative aux patients, à la santé et aux territoires", datant de 2009.

5. Association de santé mentale du $13^{\mathrm{e}}$ arrondissement (Paris).

6. Centre hospitalier public interdépartemental de psychiatrie de l'enfant et de l'adolescent.

7. Centres médicaux-psycho-pédagogiques.

\section{Références}

Azoulay, J. (2004). La psychothérapie institutionnelle, quelques repères théoriques et techniques en particulier en hôpital de jour. Revue des hôpitaux de jour et des thérapies institutionnelles, $\mathrm{n}^{\circ}$ 6, 9-11.

Barus-Michel, J. (1987). Le sujet social. Étude de psychologie sociale clinique. Paris: Dunod.

Chagnon, J.Y. (2013). Jacques Hochmann, Dans D. Drieu (dir.), 46 commentaires de textes en clinique institutionnelle (p. 99-105). Paris: Dunod.

Chaperot, C. et Celacu, V. (2008). Psychothérapie institutionnelle à l'hôpital général: négativité et continuité. L'Information psychiatrique, 84 (5). 445-453.

Crété, P. (2013). Jean Oury, Dans D. Drieu (dir.), 46 commentaires de textes en clinique institutionnelle (p. 137-143). Paris, Dunod.

Drieu, D. (2009). Adolescence en rupture et alternative thérapeutique - L'exemple d'un groupe parents d'adolescents en centre de guidance. Revue de psychothérapie psychanalytique de groupe, no 53,171-183.

Drieu, D. (2013). Introduction. Dans D. Drieu (dir.), 46 commentaires de textes en clinique institutionnelle. Paris: Dunod.

Drieu, D. (2013). René Roussillon. Dans D. Drieu (dir.), 46 commentaires de textes en clinique institutionnelle (p. 279-284). Paris: Dunod.

Delion, P. (2001). Thérapeutiques institutionnelles. EMC-Psychiatrie, 37- 930-G-10.

Delion, P. (2006). La psychothérapie institutionnelle, retour vers le futur. Rhizome, $\mathrm{n}^{\circ} 25$, 12-15.

Delion, P. (2011). Soigner la personne psychotique en institution-concepts, pratiques et perspectives de la psychothérapie institutionnelle. Paris: Dunod.

Furtos, J. (2002). Le syndrome d'auto-exclusion. Rhizome, nº 9, 15. 
Fustier, P. (1993). Les corridors du quotidien-clinique du quotidien et éducation spécialisée en institution. Paris: Dunod, 2008.

Fustier, P. (2012). L'interstitiel et la fabrique de l'équipe. Dans Nouvelle Revue de psychosociologie, 2 (14), 85-96.

Hochmann, J. (1982). L'institution mentale: du rôle de la théorie dans les soins psychiatriques désinstitutionnalisés. L'Information psychiatrique, 58 (8), 985-990.

Kaës, R. (2012). Le malêtre. Paris: Dunod.

Oury, E. et Orange, J. (2013). Guy Baillon. Dans D. Drieu (dir.), 46 commentaires de textes en clinique institutionnelle (p. 153-160). Paris: Dunod.

Oury, J. (1990). Pathique et fonction d'accueil en psychothérapie institutionnelle. Dans J. Schotte (dir.), Le contact. Bruxelles: De Boeck.

Oury, J. (2001). Psychiatrie et psychothérapie institutionnelle. Nîmes: Éditions du Champ social.

Pinel, J.-P. (2007). La construction du dispositif d'intervention à l'épreuve des mutations institutionnelles contemporaines. Dans O. Nicolle et R. Kaës (dir.), L'institution en héritage - Mythes de fondation, transmissions, transformations (p. 11-24). Paris: Dunod.

Pinel, J.-P. (2008). Emprise et pouvoir de la transparence dans les institutions spécialisées. Revue de psychothérapie psychanalytique de groupe, 51 (2), 33-48.

Robin R., (2013). Pierre Delion. Dans D. Drieu (dir.), 46 commentaires de textes en clinique institutionnelle (p. 161-166). Paris: Dunod.

Roussillon, R. (1988). Espaces et pratiques institutionnelles: Le débarras et l'interstice. Dans R. Kaës et al. (dir.), L'institution et les institutions: Études psychanalytiques (p. 157-178) Paris: Dunod.

Simon, H. (1933). La psychothérapie à l'asile. L'Hygiène mentale, $\mathrm{n}^{\circ} 1$.

Winnicott, D.-W. (1958). Processus de maturation chez l'enfant. Développement affectif et environnement. Paris: Payot, 1989.

Winnicott, D.-W. (1971). Jeu et réalité, l'espace potentiel. Paris: Gallimard, 1975. 